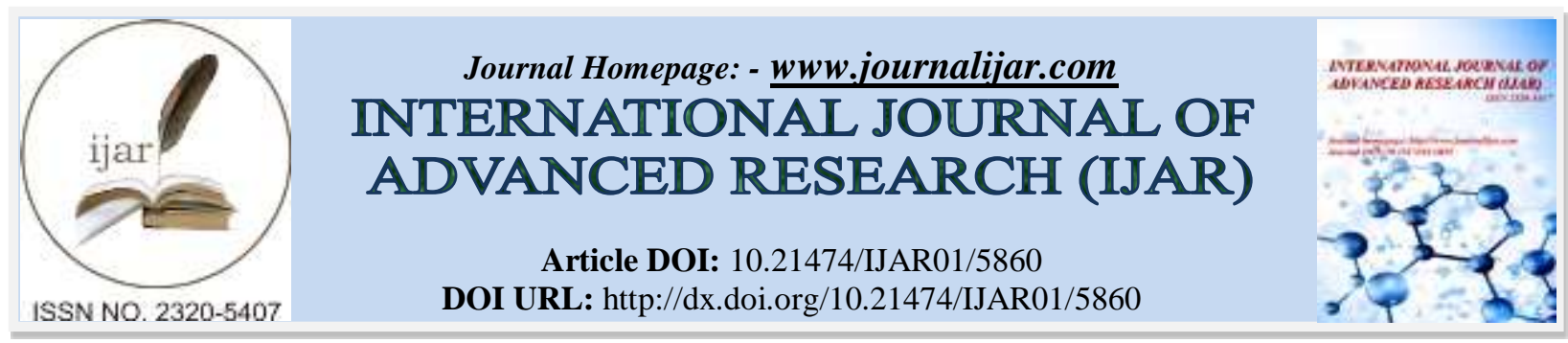

RESEARCH ARTICLE

\title{
WORD RECOGNITION OF KANNADA TEXT IN SCENE IMAGES USING NEURAL NETWORK.
}

\author{
Puneet Shetteppanavar and Aravinda Dara. \\ Department of Computer Science and Engineering, Sree Dattha Institute of Engineering and Science, \\ Ibrahimpatnam, Hyderabad, 501510, India.
}

\section{Manuscript Info}

Manuscript History

Received: 14 September 2017

Final Accepted: 16 October 2017

Published: November 2017

Key words:-

Zone wise features, Scene Images, Word

Recognition, Neural Network Classifier.

\begin{abstract}
Text Recognition in natural scene images is becoming a prominent research area and is promoting several new applications such as; car plate recognition, reading aid for the blind, tour guide systems and many more. Text recognition at word/character level is one of the key steps for development of such applications. In this work, a novel method for recognizing Kannada words in scene images is proposed. The proposed method uses zone wise horizontal and vertical profile based features. The system is efficient and insensitive to variations in size and style, noise, blur and other degradations.
\end{abstract}

Copy Right, IJAR, 2017,. All rights reserved.

\section{INTRODUCTION:-}

The recognition of text in natural scene images captured from camera inbuilt mobile phones has been current topic of interest in pattern recognition. The text in scene images is useful information for the needs and safety of the people. The scene text can be institute names, company names, building names and warning signs etc. Hence, it is necessary to recognize text in scene images useful for various applications such as; reading aid for the blind, tour guide system, traffic warning systems and many more. The recognition of scene text presents various challenges such as unusual fonts, variable lighting condition, noise, blur, variation in color, complex background, variable writing styles etc. The present work addresses the issues involved in developing an automatic method for word recognition of Kannada text in scene images.

The proposed method investigates use of zone wise horizontal and vertical profile based features for word recognition of Kannada text captured from display boards of Karnataka in India.

A few recent works have explored the problem of recognizing text in scenes [2, 5, 9, 10]. Sanjeev Satheesh, Bipin Suresh and Tao Wang [8] have addressed the problem of text detection and character recognition in scene images with unsupervised feature learning. The method achieves significant detection results, but relies on a scalable feature learning algorithm for subsequent recognition. Thus, they are not directly applicable for the challenging datasets we consider. Bodla Rakesh Babu and Manik Varma [3] proposed a method for character recognition in natural images. Although character recognition forms an essential component of text recognition, extending this framework to recognize words is not trivial as training is expensive and time consuming. Celine Mancas Thillou and Bernard Gosselin [1] describe character segmentation by recognition using Log-Gabor Filters. This dynamic segmentation uses the recognition step to give more satisfactory results and to estimate parameters of Log-Gabor filters. These filters are very appropriate for character segmentation by using as central frequency the inverse of estimation of character thickness and as bandwidth a dynamic choice to handle all unexpected variations in natural scene 
characters. Natural scene images have numerous and various degradations and to handle these issues, it is very important to link consecutive steps together to take dynamic decisions.

The proposed approach is more closely related to those in [4, 6, 7], which address the problem of word recognition of Kannada text in scene images using neural network. On one hand, these methods recognize text with a significant accuracy, but on the other hand, their recognition results leave a lot to be desired. Note that the challenges of this task are evident from the best published accuracy of only $85.53 \%$ on the scene text dataset [4]. The probabilistic approach we propose in this paper achieves an accuracy of over $97.17 \%$ under identical experimental settings.

The remainder of the paper is organized as follows. In Section 2 we present our proposed methodology. We provide results on two public datasets and compare our method to related work in Section 3. Implementation details are also given in this section.

\section{Proposed Methodology:-}

The proposed method uses zone wise horizontal and vertical profile based features for word recognition. The method consists of various phases such as, pre-processing and segmentation, feature extraction, training neural network and word recognition model. Figure 1 shows the block diagram of the proposed model. The detailed description of each is given in the following subsections.

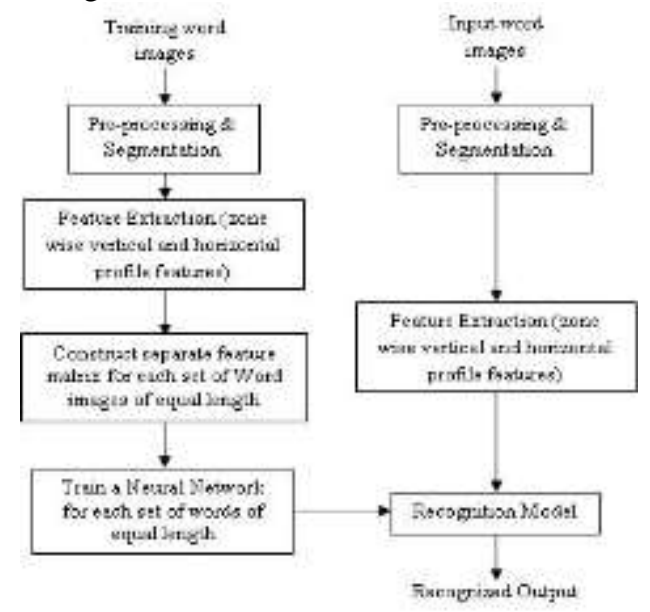

Fig. 1:- Block Diagram of Proposed Model.

\section{A. Pre-processing and Segmentation}

The scene text images have issues like lighting effects, shadowing, blur, color degradation and size etc. The purpose of this phase is to make the images to be of standard size and remove complex backgrounds easier for further processing. Pre-processing and segmentation procedure consists of several steps, which are as detailed below;

\section{Binarization}

The input word image is converted into binary image that has only two possible values for each pixel represented by either 0 or 1 . Every word image is resized to fixed size based on length.

\section{Segmentation}

In this phase, the word image is portioned into individual character images. The goal of segmentation is to simplify an image that is more meaningful and easier to analyze. This segmentation step is crucial for the word recognition: any error directly reduces the recognition accuracy of the system.

\section{Thinning}

Thinning refers to the process of reducing the width of a line like object from many pixels wide to just single pixel. This process can remove irregularities in letters and in turn, makes the recognition algorithm simpler because they only have to operate on a character stroke, which is only one pixel wide. 


\section{Bounding Box Generation}

Before analysing any character of the word image, it is important to identify the (pixel) boundaries of that character. Thus, a bounding box is generated around the image.

\section{B. Feature extraction}

Features are extracted from the segmented pre-processed characters of the word image (i.e. word image may be 2,3,4,5 or 6 character image). Each character image is divided into 6 vertical zones and 6 horizontal zones, where size of each horizontal zone is $5 \times 30$ and the size of each vertical zone is $30 \times 5$. Then sum of all pixels in every zone is determined as a feature value. Totally 12 features are obtained from each character stored in feature vector. Feature vector is shown in the equation (1).

$$
C_{f v}=\left[V_{f 1} \ldots . V_{f 6} H_{f 1} \ldots . H_{f 6}\right]
$$

The feature vector for word image consisting of " $\mathrm{N}$ " number of characters is depicted in equation (2).

Where $1<=\mathrm{i}<=\mathrm{N}$ and $2<=\mathrm{N}<=6$

$$
\mathrm{W}_{\mathrm{FV}}=\left[\mathrm{C}_{\mathrm{FV}}^{\mathrm{I}}\right]
$$

$C_{f v}^{i}$ is the Feature Vector of the ith character of the word image.

$W_{f v}$ is the Word feature vector comprising feature of all characters.

\section{Neural Network Training}

The Feed Forward Neural Network is used for training the network. The features generated from the training database are used to train the model. The Neural Network that is used for the training has 36 input neurons, 38 hidden neurons and 6 output neurons. Figure 2 shows the Neural Network model used for training.

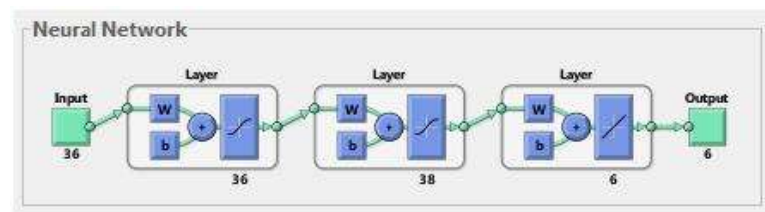

Fig. 2:- Neural Network Model.

D. Word Recognition Model.

Test image is processed to obtain zone wise horizontal and vertical profile based features, which are further fed to Neural Network for recognition.

\section{EXPERIMENTAL RESULTS AND DISCUSSIONS:-}

The dataset is collected from government office display boards, traffic boards and boards on various buildings in Karnataka. The dataset consists, 750 images of Kannada words. The proposed methodology for word recognition system has been evaluated for several samples dealing with various issues. The method achieves recognition accuracy of $97.17 \%$. The system is efficient and insensitive to the variations in size and font, noise, blur and other degradations.

\section{A. Sample Kannada Word Image containing Blur}

The word image that is selected from the database is given in figure 3. The image has several challenges like unusual fonts and size, blur etc. Pre-processing step is performed to make the images to be of standard size, remove complex backgrounds and makes them easier for further processing in word recognition. In Pre-processing step the color image is converted into gray scale image, then into binary image. The binary image is resized and then applying thinning process and bounding box. Then features for each word are extracted and then testing is performed.

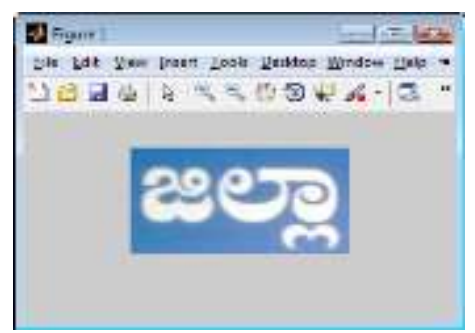

Figure 3:- Word Image jilla 
The resulting gray scale image and resized binary image is shown in figure 3.1 and figure 3.2 respectively.

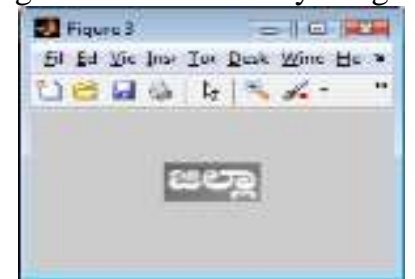

Figure 3.1 Gray Scale Image.

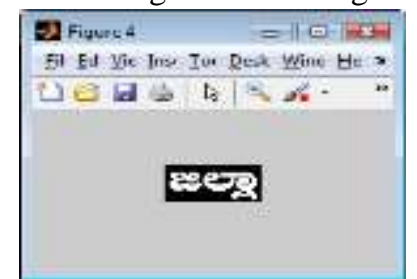

Figure 3.2 Resized Binary Image.

The segmentation process is shown in figure 3.3.
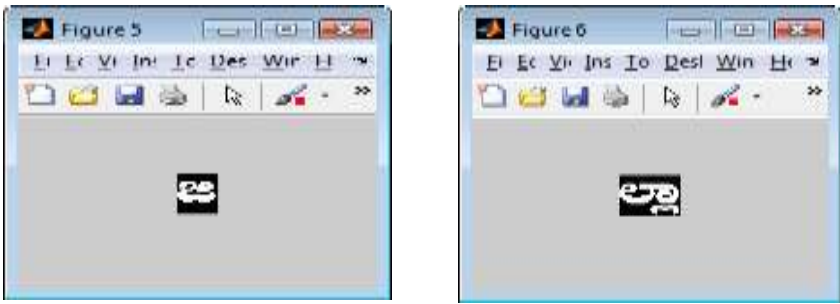

Figure 3.3:- Segmentation Process (Word Segmented into Characters).

The resulting thinned image and thinned image with bounding box is shown in figure 3.4 and figure 3.5 respectively.
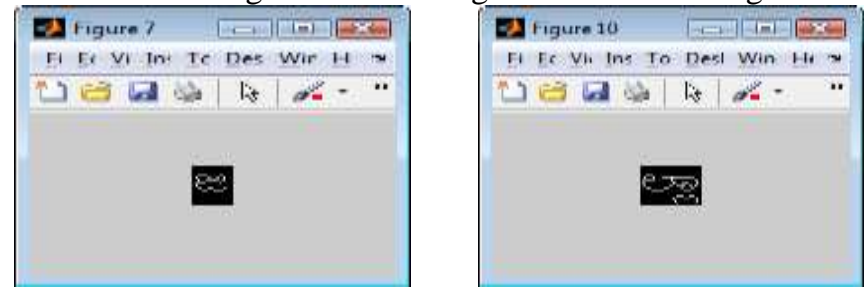

Figure 3.4:- Thinned Images.
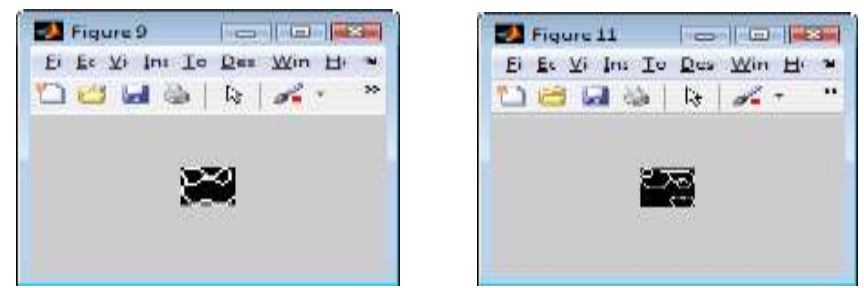

Figure 3.5:- Thinned Images with Bounding Box.

The zone wise vertical and horizontal profile features are as follows.

$\begin{array}{llllll}57 & 34 & 42 & 31 & 36 & 34 \\ 47 & 52 & 49 & 10 & 15 & 61 \\ 24 & 12 & 15 & 35 & 34 & 30 \\ 48 & 36 & 28 & 11 & 17 & 10\end{array}$

The method is able recognize blurred word image correctly. The recognized pattern is given in table 1 .

TABLE 1:- OUTPUT PATTERN FOR WORD IMAGE JILLA.

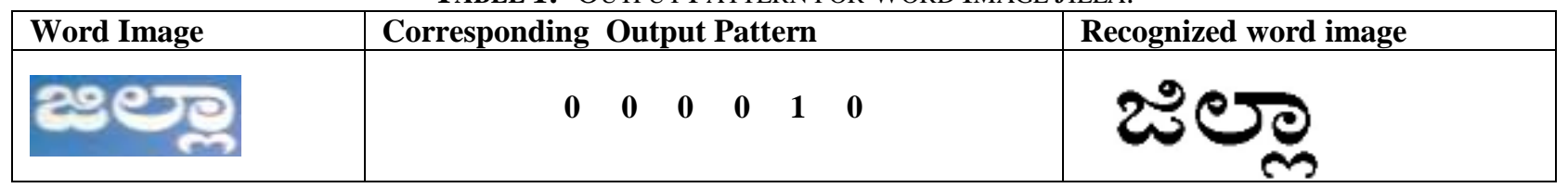


B. Sample Kannada Word Image with Variable Font Size

The word image that is selected from the database is given in figure 4. The image has challenge like variable font size. The resulting gray scale image is shown in figure 4.1.

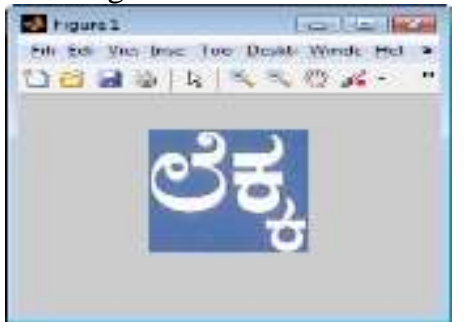

Figure 4:- Word Image lyakka.

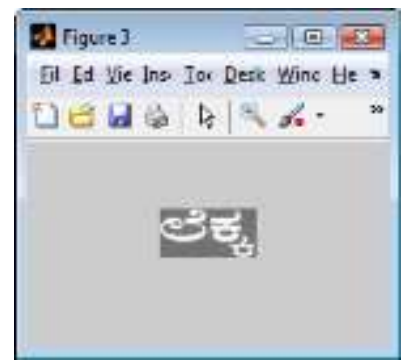

Figure 4.1 Gray Scale Image.

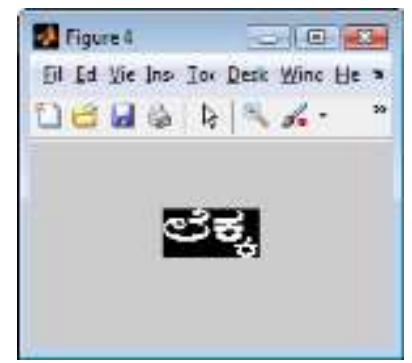

Figure 4.2 Resized Binary Image.

The resized binary image and segmentation process is shown in figure 4.2 and figure 4.3 respectively.
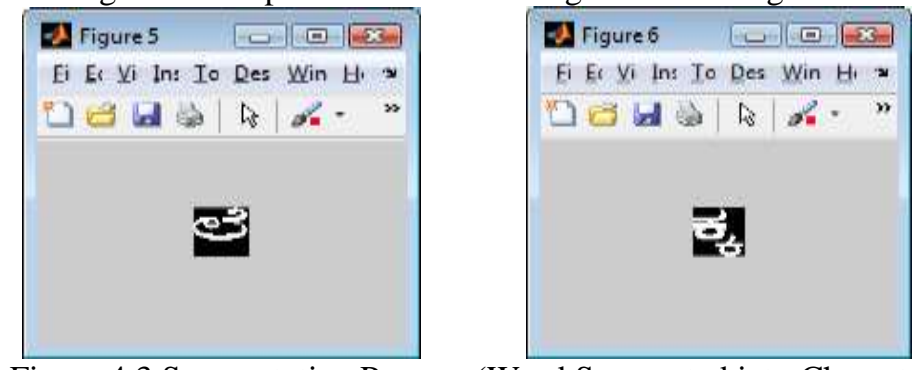

Figure 4.3 Segmentation Process (Word Segmented into Characters).

The thinned and thinned image with bounding box is shown in figure 4.4 and figure 4.5 respectively.
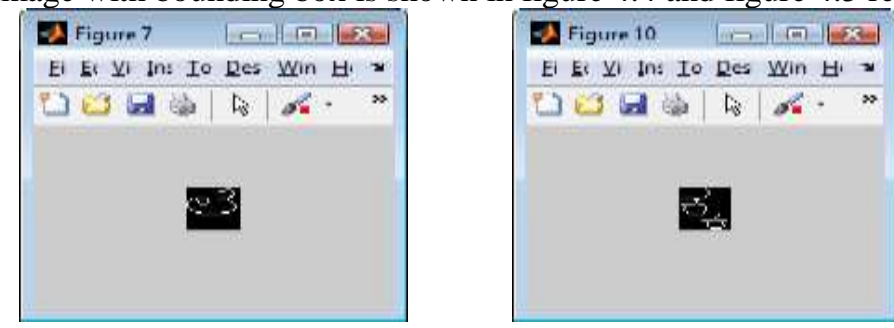

Figure 4.4 Thinned Images
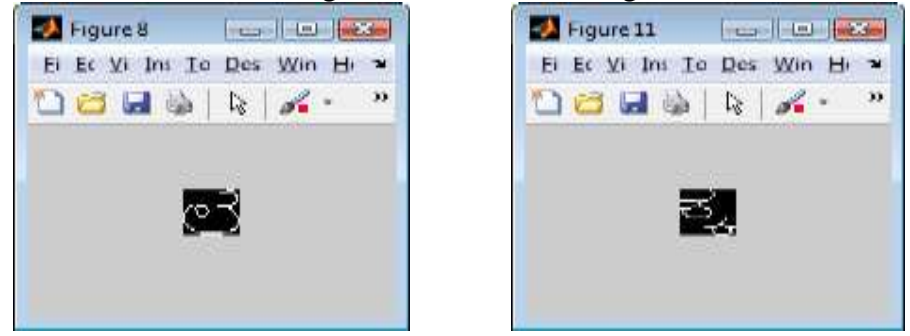

Figure 4.5 Thinned Images with Bounding Box. 
The zone wise vertical and horizontal profile features are as follows.

$\begin{array}{llllll}21 & 20 & 22 & 15 & 24 & 36 \\ 16 & 7 & 46 & 18 & 10 & 41 \\ 23 & 18 & 23 & 23 & 16 & 13 \\ 5 & 32 & 20 & 20 & 21 & 18\end{array}$

The method is able recognize word correctly in the presence of variable font size. The recognized pattern is given in table 2 .

TABLE 2:- OUTPUT PATTERN FOR WORD IMAGE LEKKA.

\begin{tabular}{|l|lllllll|}
\hline Word Image & Corresponding output pattern & Recognized Word image \\
\hline & 0 & 0 & 0 & 1 & 1 & 0 & \\
\hline
\end{tabular}

C. Sample Kannada Word Image with Dark Background

The word image that is selected from the database is given in figure 5. The image has challenge like dark background. The resulting gray scale image and resized binary image is shown in figure 5.1 and figure 5.2 respectively.

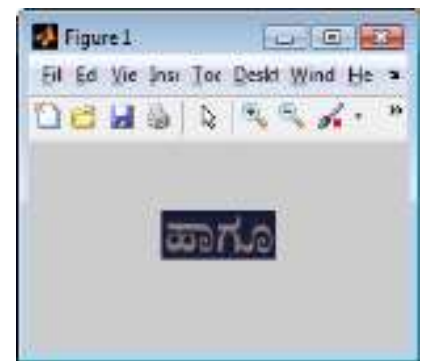

Figure 5:- Word Image hagu

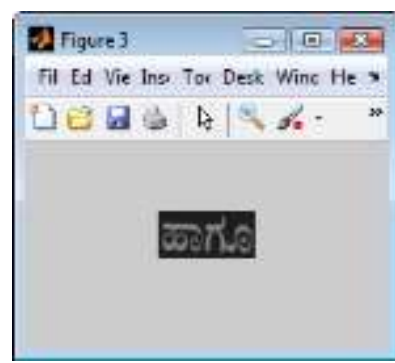

Figure 5.1 Gray Scale Image.

The segmentation process is shown in figure 5.3.

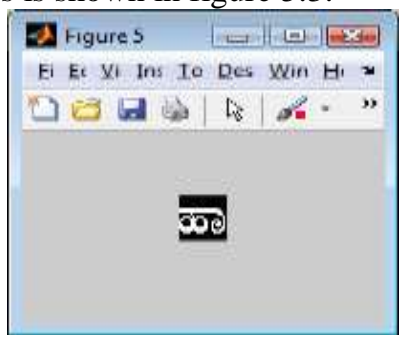

Figure 5.4 Segmentation Process (Word Segmented into Characters).

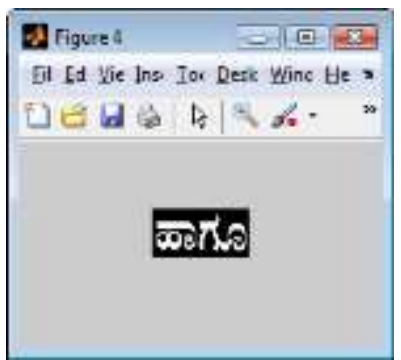

Figure 5.2 Resized Binary Image

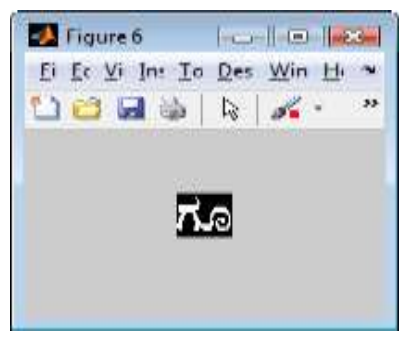


The thinned image is shown in figure 5.5.
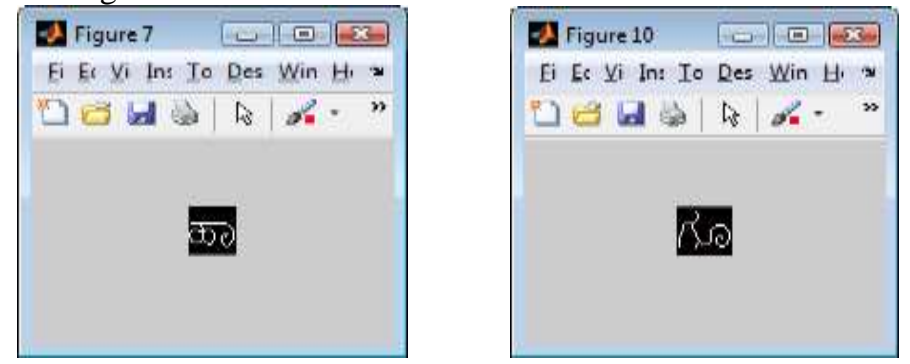

Figure 5.5 Thinned Images.

Thinned image with bounding box is shown in figure 5.6.
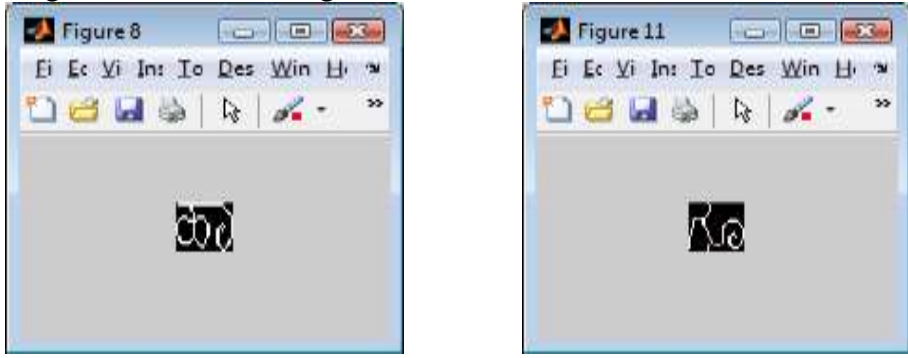

Figure 5.6 Thinned Images with Bounding Box.

The zone wise vertical and horizontal profile features are as follows.

$\begin{array}{llllll}36 & 49 & 30 & 34 & 42 & 36 \\ 57 & 27 & 35 & 38 & 44 & 26 \\ 22 & 16 & 23 & 16 & 28 & 24 \\ 5 & 10 & 32 & 23 & 32 & 27\end{array}$

The method is able recognize word correctly in the presence of dark background. The recognized pattern is given in table 5 .

TABLE 3:- OUTPUT PATTERN FOR WORD IMAGE HAAGU.

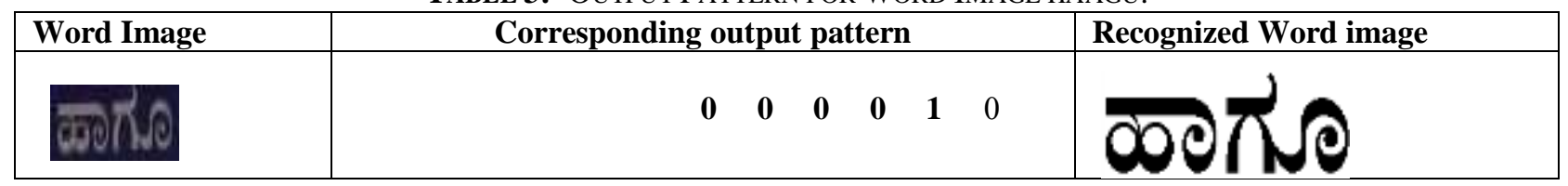

D. Sample Kannada Word Image containing Noise

The Word image that is selected from the database is given in figure 6. The image has challenge like noise. The resulting gray scale image and resized binary image are shown in figure 6.1 and figure 6.2 respectively.

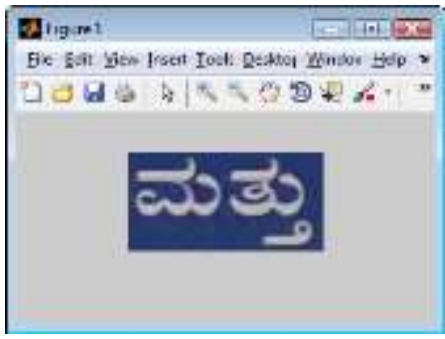

Figure 6 Word Image mattu 


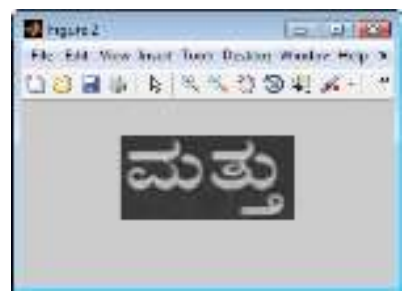

Figure 6.1 Gray Scale Image.

The segmentation process is shown in figure 6.3.

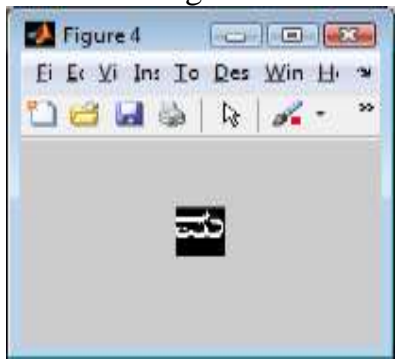

Figure 6.3 Segmentation Process (Word Segmented into Characters).

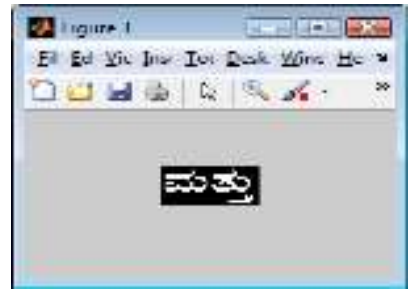

Figure 6.2 Resized Binary Image.

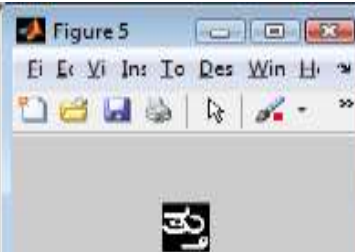

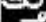

The thinned image is shown in figure 6.4

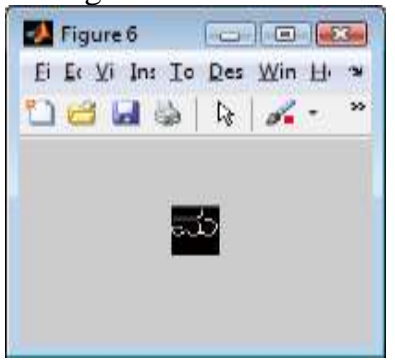

Figure 6.4 Thinned Images

Thinned image with bounding box is shown in figure 6.5
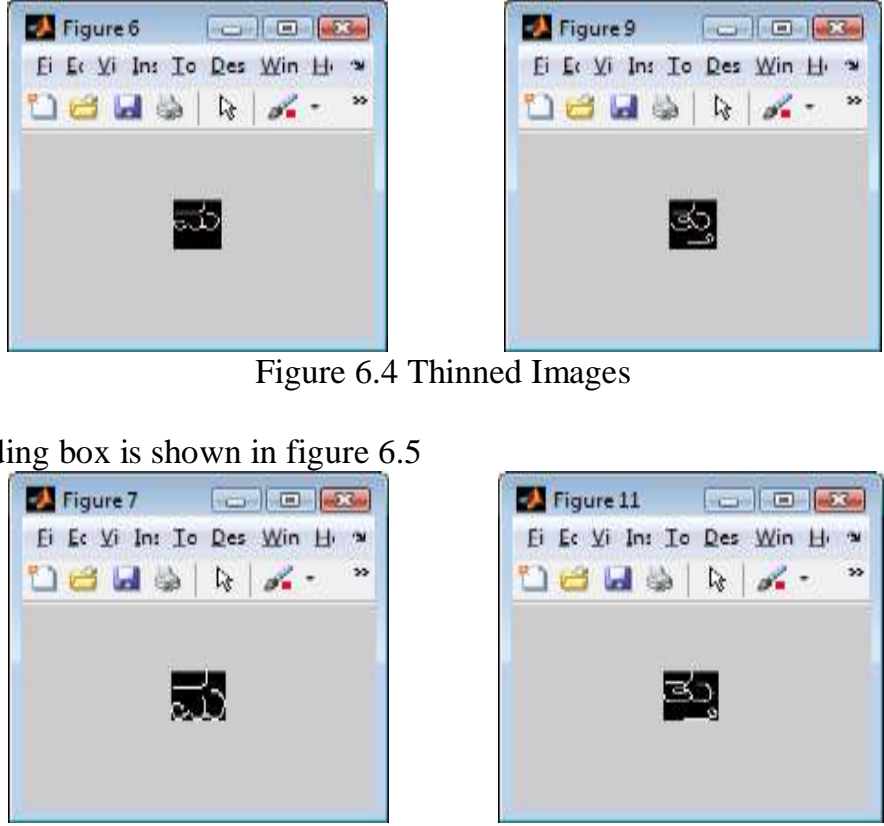

Figure 6.5 Thinned Images with Bounding Box.

The zone wise vertical and horizontal profile features are as follows.

$\begin{array}{llllll}29 & 24 & 19 & 37 & 15 & 23 \\ 5 & 23 & 28 & 15 & 23 & 53 \\ 12 & 20 & 26 & 19 & 20 & 22 \\ 5 & 26 & 27 & 31 & 6 & 24\end{array}$

The method is able recognize word correctly in the presence of dark background. The recognized pattern is given in table 6.

TABLE 4:- OUTPUT PATTERn FOR WORD IMAGE MATTU.

\begin{tabular}{|c|c|c|}
\hline Word Image & Corresponding output pattern & Recognized Word image \\
\hline & $\begin{array}{lllllll}0 & 0 & 0 & 1 & 0 & 0\end{array}$ & \\
\hline
\end{tabular}


Though, scene images contain certain challenges like variation in size and font, noise, blur and other degradations, the proposed method works efficiently.

The experiment is performed on 750 Word Images, where in 600 word samples are used for training and testing is done on 750 word samples. The following table 1 shows overall performance of the system.

TABLE 5:- SYSTEM PERFORMANCE

\begin{tabular}{|l|l|l|l|l|}
\hline $\begin{array}{l}\text { Trained } \\
\text { Samples }\end{array}$ & Tested Samples & $\begin{array}{l}\text { Correctly Recognized } \\
\text { Samples }\end{array}$ & $\begin{array}{l}\text { Falsely Recognized } \\
\text { Samples }\end{array}$ & $\begin{array}{l}\text { Recognition } \\
\text { Accuracy }\end{array}$ \\
\hline 600 & 750 & 728 & 22 & $97.17 \%$ \\
\hline
\end{tabular}

\section{CONCLUSION:-}

This work strives toward a novel methodology that aids pre-processing, segmentation and recognition of Kannada Words from camera based images. The proposed methodology is based on zone wise horizontal and vertical profile based features and neural network as a classifier for Kannada Word Recognition. The system works in two phases training phase and testing phase. Exhaustive experiments are done for analysis of zone wise horizontal and vertical profile based features.

The system successfully processes camera based images having challenges like variable lightning condition, noise, blur, unusual fonts etc. The methodology is tested with 750 samples and gives recognition accuracy of $97.17 \%$. The method can be extended for word recognition considering new set of features and classification algorithms.

\section{REFERENCES:-}

1. Celine Mancas Thillou, Bernard Gosselin, "Character Segmentation by Recognition using Log-Gabour Filters", The 18th International Conference on Pattern Recognition, vol 2, PP 901 - 904, 18 September 2006.

2. Zora Saidane and Christophe Garcia, "Automatic Scene Text Recognition using a Convolution Neural Network", Proceedings of the Second International Workshop, 2007.

3. Bodla Rakesh Babu, Manik Varma, "Character Recognition in Natural Images", International Conference on Computer Vision Theory and Applications (VISAPP), 2008.

4. Sangame S.K., Ramteke R.J., Rajkumar Benne, "Recognition of isolated handwritten Kannada vowels", Advances in Computational Research (ISSN) Vol 1, No 2, 2009.

5. Jerod J. Weinman, Erik Learned-Miller, "Scene Text Recognition Using Similarity and a Lexicon with Sparse Belief Propagation", IEEE Transactions on Pattern Analysis and Machine Intelligence, Vol 31, No 10, October 2009.

6. Rokus Arnold, Poth Miklos, "Character Recognition Using Neural Networks", 11th IEEE International Symposium on Computational Intelligence and Informatics, 18th -20th November 2010.

7. Leena Ragha, M Sasikumar, "Adapting Moments for Handwritten Kannada Kagunita Recognition”, Second International Conference on Machine Learning and Computing, 2010 IEEE.

8. Sanjeev Satheesh, Bipin Suresh, Tao Wang, "Text Detection and Character Recognition in Scene Images with Unsupervised Feature Learning”, International Conference on Document Analysis and Recognition (ICDAR), 2010 IEEE.

9. Heejin Chung, Kue-Hwan Sihn, Sooncheol Hong, Hyo Jung Song, "Scene Text Recognition System using Multigrain Parallelism", The 8th Annual IEEE Consumer Communications and Networking Conference, 2011 IEEE.

10. Kai Wang, Boris Babenko and Serge Belongie, "End-to-End Scene Text Recognition", IEEE International Conference on Computer Vision (ICCV), PP 1457 - 1464, 2011.

11. U. V. Marti, H. Bunke, "Text Line Segmentation and Word Recognition in a System for General Writer Independent Handwriting Recognition", Sixth International Conference on Document Analysis and Recognition (ICDAR), PP 159 - 163, 10-13 Sep 2001.

12. Jeord J. Weinman Erik Learned-Miller and Allen Hanson, "A Discriminative Semi-Markov Model for Robust Scene Text Recognition", International Conference on Pattern Recognition (ICPR), PP 1 - 5, 8-11 Dec 2008.

13. Xavier Domont, Martin Heckmann, "Word Recognition with a Hierarchical Neural Network", Proceedings of the international Conference on Advances in nonlinear speech processing (ISBN), 2006. 
14. B. Vijay Kumar, A. G. Ramakrishnan, "Radial Basis Function and Subspace approach for Printed Kannada Text Recognition", IEEE Conference on Acoustics, Speech, and Signal Processing (ICASSP), PP V-321-4, Vol 5, 17-21 May 2004.

15. B.M. Sagar, Dr. Shobha G, Dr. Ramakanth Kumar, "Character Segmentation Algorithms for Kannada Optical Character Recognition", Proceedings of the 2008 International Conference on Wavelet Analysis and Pattern Recognition, Hong Kong (ICWAPR), Vol-1, PP 339 - 342, 30-31 Aug. 2008.

16. Rui Chen, Cao Beijun, Mohammad Reza Asharif, Katsumi Yamashita, "Character Recognition Algorithm based on the Central Location and Polar Projection", 2nd International Congress on Image and Signal Processing (CISP), PP 1-4, 17-19 October 2009.

17. Lukas Neumann and Jiri Matas, "A Method for Text Localization and Recognition in Real-Word Images", Published at the 10th Asian Conference on Computer Vision, Queenstown, New Zealand. 8-12 November 2010.

18. Chetan S, "Character Recognition using Artifical Neural Networks", Department of Computer Science \& Engineering, NIT Calicut, 2006.

19. B.M. Sagar, Dr. Shobha G, Dr. Ramakanth Kumar, "Complete Kannada Optical Character Recognition with Syntactical Analysis of the script", Proceedings of the 2008 International Conference on Computing, Communication and Networking (ICCCNET), PP 1 - 4, 18-20 December 2008.

20. Annika Kuhl, Tele Tan, Svetha Venkatesh, "Model-based Character Recognition in Low resolution", 15th IEEE International Conference on Image processing (ICIP), PP 1001 - 1004, 12-15 October 2008. 